\title{
Estimating rate uncertainty with maximum likelihood: differences between power-law and flicker-random-walk models
}

\author{
John Langbein
}

Received: 14 November 2011 / Accepted: 15 March 2012 / Published online: 11 April 2012

(C) The Author(s) 2012. This article is published with open access at Springerlink.com

\begin{abstract}
Recent studies have documented that global positioning system (GPS) time series of position estimates have temporal correlations which have been modeled as a combination of power-law and white noise processes. When estimating quantities such as a constant rate from GPS time series data, the estimated uncertainties on these quantities are more realistic when using a noise model that includes temporal correlations than simply assuming temporally uncorrelated noise. However, the choice of the specific representation of correlated noise can affect the estimate of uncertainty. For many GPS time series, the background noise can be represented by either: (1) a sum of flicker and random-walk noise or, (2) as a power-law noise model that represents an average of the flicker and random-walk noise. For instance, if the underlying noise model is a combination of flicker and random-walk noise, then incorrectly choosing the power-law model could underestimate the rate uncertainty by a factor of two. Distinguishing between the two alternate noise models is difficult since the flicker component can dominate the assessment of the noise properties because it is spread over a significant portion of the measurable frequency band. But, although not necessarily detectable, the random-walk component can be a major constituent of the estimated rate uncertainty. None the less, it is possible to determine the upper bound on the random-walk noise.
\end{abstract}

Keywords Rate $\cdot$ Uncertainty $\cdot$ Bias $\cdot$ Least squares

J. Langbein ( $ه)$

US Geological Survey, Earthquake Science Center,

345 Middlefield Rd., Menlo Park, CA 94025, USA

e-mail: langbein@usgs.gov

\section{Introduction}

One of the key products derived from GPS observations is the velocity and its uncertainty for a suite of sites distributed over a geographical region. From the velocities, a first-order inference is whether there is detectable deformation within the network of GPS monuments. For instance, in the area straddling the Mississippi River in the central US, there is a debate about whether there is significant deformation associated with the New Madrid Seismic zone, where, on one hand, Calais and Stein (2009) argue that within the uncertainty of the data, there is no significant deformation; but, on the other hand, Frankel and Smalley (2011) detect some deformation that is consistent with creep at depth on the fault that last ruptured in the sequence of $\sim M 7$ earthquakes in 1811-1812 (Hough and Page 2011). To understand the differences in the inference for detectable deformation, a critical examination of the ingredients and assumptions that go into estimating the uncertainty in rate is required. As I shall demonstrate, the choice of a model that characterizes the background noise of GPS time series can lead to a factor of two difference in the rate uncertainty, which, if applied to a region with low deformation rate, could skew ones interpretation of the mechanism for deformation. Lacking a robust method for characterizing the noise in the data at its longest period, I provide here one means of obtaining an upper bound for noise where it has the most impact for calculating the rate uncertainty.

It is now widely recognized that time series of positions derived from GPS measurements have temporal correlations for both global networks (e.g. Mao et al. 1999; Williams et al. 2004; Amiri-Simkooei et al. 2007; Santamaría-Gómez et al. 2011) and regional networks (e.g. Zhang et al. 1997; Williams et al. 2004; Beavan 2005; Langbein 2008). These temporal correlations are modeled as a combination of white and colored noise. In the frequency domain, the white noise (WN) 
is frequency independent, while the colored noise is represented as a function of $1 / f^{n}$. In special cases, when $n=1$, the noise model is termed flicker (FL), and, when $n=2$, the noise model is termed random walk (RW); otherwise, it is termed power-law noise (PL). The difference in definition between global and regional networks has to do with the network's spatial extent and the data processing methods. Global networks typically span more than one continent while regional networks span an area that is smaller than a continent. In addition, for sites that comprise a regional network, the time series of position change exhibit commonmode displacements, and these can be removed by some form of stacking and averaging over a suite of time series (e.g. Wdowinski et al. 1997). By removing the common-mode signal, the RMS scatter in the time series tends to be less for the regional networks than for the global networks.

For data from regional GPS networks, which is the subject discussed in this paper, the indices estimated for the PL model range between 1 and 2 (Williams et al. 2004; Langbein 2008). But Langbein (2008) also demonstrated that an alternate noise model could be a combination of FL and RW noise (FLRW). As demonstrated below, the choice of model between PL and FLRW has a significant impact on the estimated uncertainty in rate even though, through maximum likelihood estimation (MLE), either noise model successfully characterizes the background noise.

Other than typically obtaining more conservative estimates of the rate uncertainty, the FLRW model is attractive since one could claim that the constituent flicker and random-walk terms each have a physical basis. In part, this argument is based upon logical deduction, since it is difficult to unambiguously parse GPS noise into specific, physical mechanisms. Ultra-high precision data from long-baseline strainmeters (Wyatt 1982, 1989; Agnew 1992), borehole strainmeters (Johnston and Linde 2002), and creepmeters (Langbein et al. 1993) all contain a strong component of random-walk noise. This noise vastly exceeds the precision and stability of the transducer and is due to random fluctuations in the local, crustal volume that surrounds the interface of the instrument to the Earth. Unlike GPS with perhaps $0.1 \mathrm{~mm}$ sensitivity, the sensitivity is of the order $0.01 \mathrm{~mm}$ for creep and $10^{-4} \mathrm{~mm}$ for long-baseline strain. Like the ultra-precise instrumentation, GPS receivers are coupled to the Earth's surface through its monuments and these monuments are prone to the same random fluctuations that are best characterized as RW noise. Subtracting a RW component from a background noise model of either PL or FLRW leaves a residual noise model that is closer to a combination of flicker and white noise. Therefore, the logical deduction is that the residual FL and WN is due to the GPS system, including the electronics of the receiver and satellites, the paths between the satellites and the receiver, and the models used to adjust the path length.
The calculation of rate uncertainty is provided in both Williams (2003) and Langbein (2004). If $A$ is the design matrix relating the observations to a model vector $x$, where one of the model components is rate and its corresponding column of $A$ is made up of the time of each observation, and $C$ is the data covariance, then the variance of the model vector is $\left[A^{t} C^{-1} A\right]^{-1}$. The data covariance is constructed using the specified values of white and power-law noise using the method outlined in both Williams (2003) and Langbein (2004).

Figure 1 illustrates two problems with estimating the rate uncertainty. First, while the PL and the FLRW noise
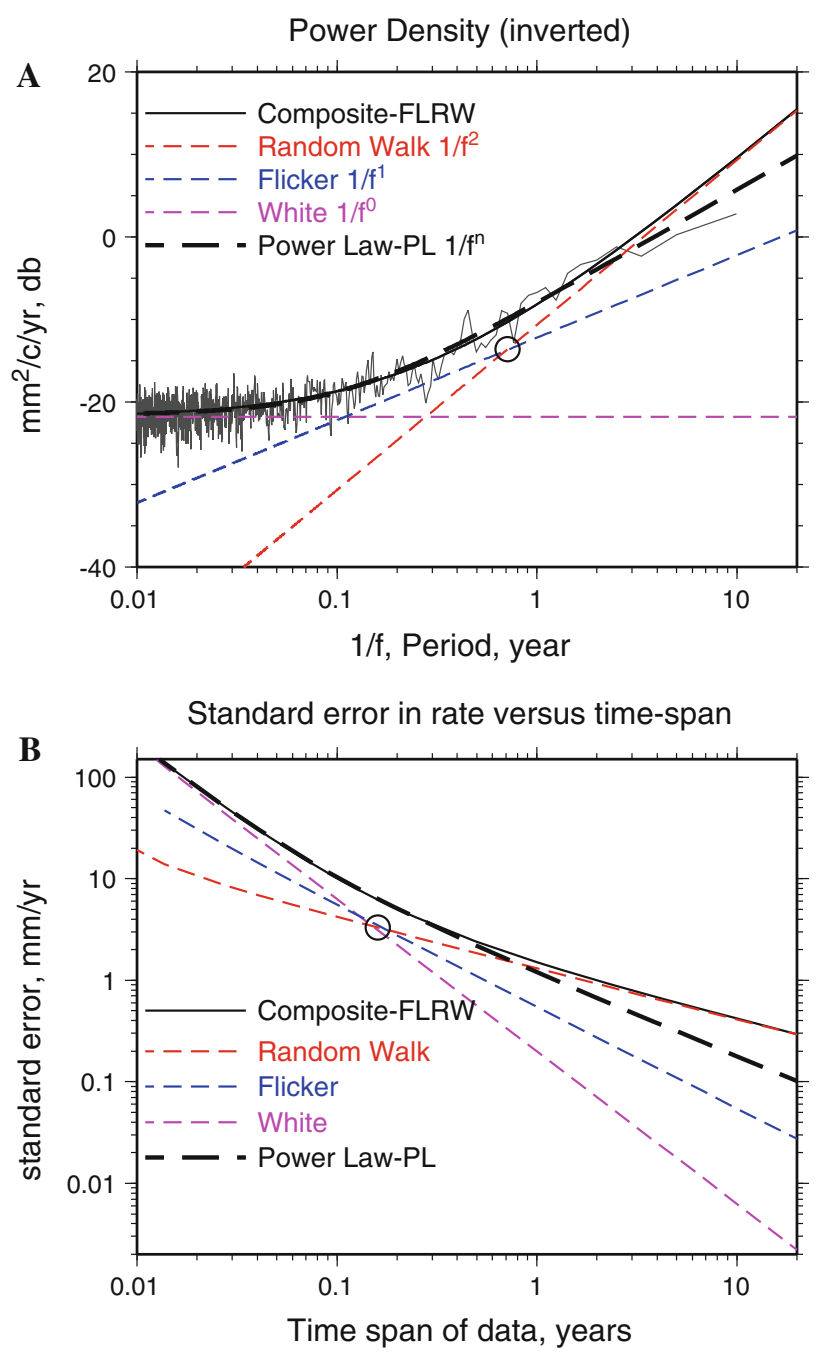

Fig. 1 Power density and rate-uncertainty spectra for noise models of GPS data. The noise model is representative of GPS data for sites for which common-mode signals have been removed. That model consists of $1.1 \mathrm{~mm}$ white, $1.9 \mathrm{~mm} /$ year $^{0.25}$ flicker, and $1.3 \mathrm{~mm} /$ year $^{0.5}$ randomwalk noise. a Power density as a function of period is shown. In gray, the spectra from a 40-year long synthetic time series shows the variability in estimates of power density. b Uncertainty in rate is shown as a function of length of time series. Indicated by circles, in $\mathbf{a}$ and $\mathbf{b}$, crossover between flicker and random-walk noise for rate uncertainty is about one quarter of the crossover in power spectrum 
have nearly identical power spectra for periods of less than 10 years, the estimated rate uncertainty deviates significantly between the two models for time spans in excess of 1 year. The power spectrum estimated from simulated data having FLRW noise illustrates the variability in estimating the spectrum as it deviates by $5 \mathrm{db}$, which is equivalent to the maximum deviation between the FLRW and PL model of noise. Even though either the PL or FLRW is a satisfactory representation of the background noise of the data, the choice of one over the other will affect the calculated rate uncertainty. Thus, the rate uncertainty could be biased either too high or too low depending upon the true underlying noise model.

The second problem is independently resolving both the FL and RW amplitudes and the impact of RW noise upon calculating the rate uncertainty, assuming that the FLRW model is the correct choice. Figure 1a shows for the periods between 0.16 and 0.71 years (or 60 and 260 days indicated by circles in Fig. 1) that the power level for RW noise is less than the FL noise level. Yet, when the rate uncertainty is computed for the FLRW model over these periods, the effect of the RW noise dominates. Thus, although RW noise is not necessarily detectable, its presence should be acknowledged as it impacts the rate uncertainty.

This paper examines both these problems using examples. In Sect. 2, the bias in rate uncertainty is explored through simulation. Section 3 offers suggestions to the problem of underestimating the RW noise component, and, importantly, provides a method to determine the upper bound of the RW noise. Computations are based upon the MLE methods using algorithms presented by Langbein (2004) and Williams et al. (2004).

\section{Bias in estimating rate uncertainty}

The problem of estimating the rate uncertainty based upon the choice of noise model is explored through simulations, and those results are shown in Figs. 2 and 3. The simulations use a background noise model consisting of $1.1 \mathrm{~mm}$ $\mathrm{WN}$ and $1.3 \mathrm{~mm} /$ year $^{0.5} \mathrm{RW}$ noise. In addition, either 0 or $1.9 \mathrm{~mm} /$ year $^{0.25}$ FL noise is added to the simulated time series. The power spectrum of the resulting FLRW noise is shown in Fig. 1a. For the simulations, several intervals were considered ranging from 1 to 20 years. For each simulated time series, a noise model consisting of PL was fit to the data using MLE techniques. The power-law amplitude, its index, white-noise amplitude, the estimated rate uncertainty, and the logarithm of the likelihood were saved. Likewise, a FLRW noise model was fit to the same time series, and the amplitudes of the FL and RW, white-noise amplitude, the estimated rate uncertainty, and the logarithm of the likelihood were also saved. For each length, 200 simulations were done and some of the statistics for the results are shown in Figs. 2 and 3.
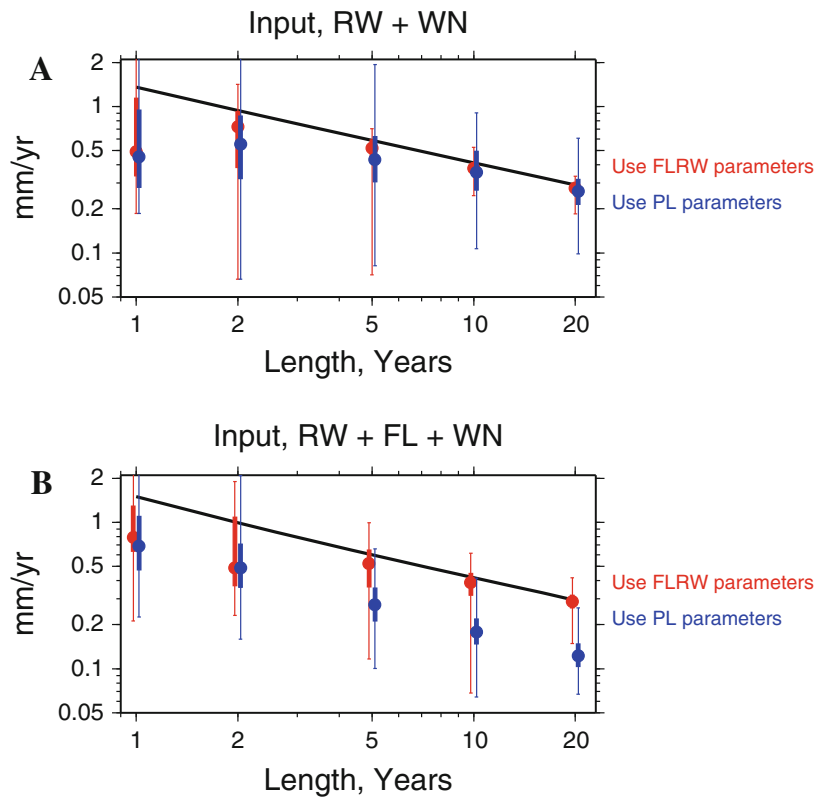

Fig. 2 Distribution of estimated rate uncertainty using either a PL model (blue) or a FLRW model (red). a Underlying time series is random walk plus white noise. b Underlying model is FLRW plus white noise. The black line is rate uncertainty for the underlying time series as a function of time-series length. The solid dots are the median estimated rate uncertainty from 200 simulations. The thick bars are the interquartile range of estimated rate uncertainty, while the thin lines are the total range of estimated rate uncertainty from simulations

In the case where the underlying noise model is RW plus $\mathrm{WN}$, the statistics of the rate uncertainty are similar independent of whether FLRW or PL is specified (Fig. 2a). For example, with a length of 1 year, specifying either FLRW or PL noise yields a rate uncertainty of about $0.5 \mathrm{~mm} /$ year. However, the true rate uncertainty is closer to $1.5 \mathrm{~mm} /$ year (black line). Although the range of the estimated uncertainty includes the true uncertainty, the central tendency is that the MLE algorithm will underestimate the rate uncertainty. The smaller rate uncertainty can be understood either by examination of the underlying power spectrum (red and magenta lines in Fig. 1) or the distribution of the estimated power-law index in Fig. 3. In the power spectrum, the cross over where RW exceeds WN is 0.3 year, which is a sizable fraction of the 1-year long interval of the simulations. Consequently, there are not enough data to resolve the longer-period deviations as the estimated index of PL noise ranges between 0 and 2.5 with a sizable cluster with $n<0.5$ (Fig. 3a).

However, as the length of the data set becomes longer, $\geq 5$ years, then estimated rate uncertainty is nearly equal to the uncertainty in the underlying data independent of the specified noise model for the MLE algorithm (Fig. 2a). The distribution of power-law indices for the longer intervals clusters around 2, in agreement with the underlying RW noise (Fig. 3a). 

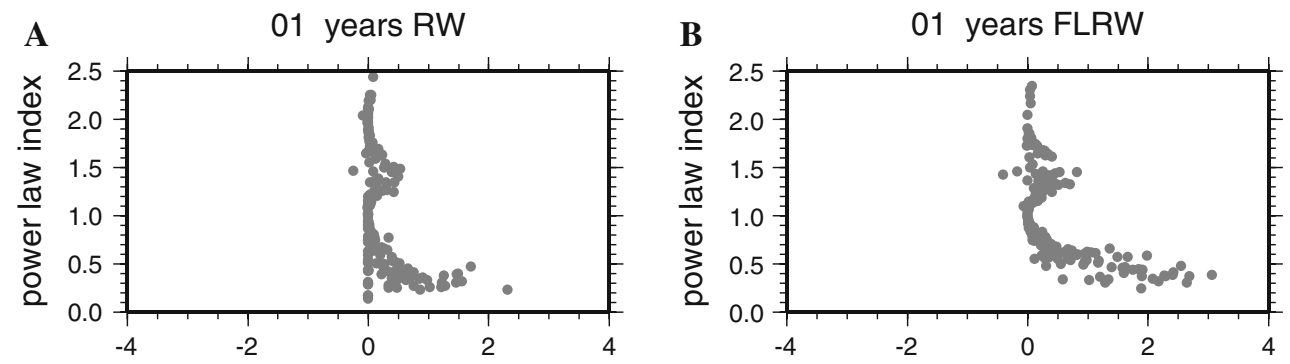

02 years RW

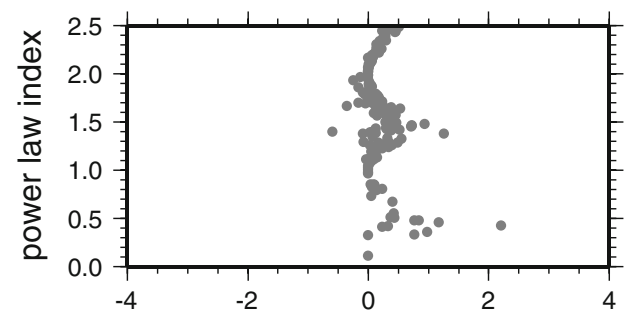

02 years FLRW

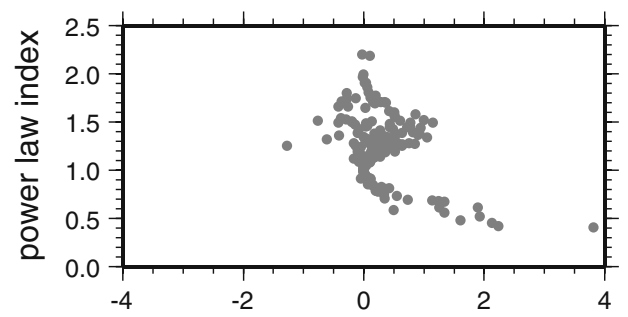

05 years RW

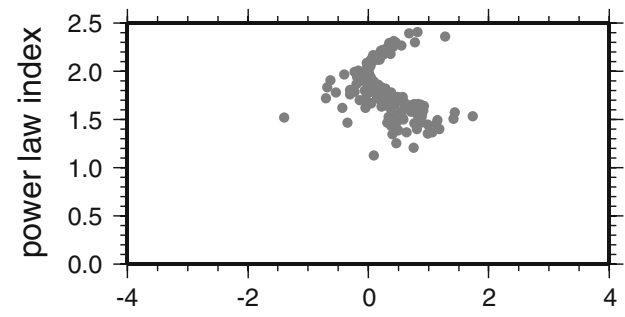

05 years FLRW

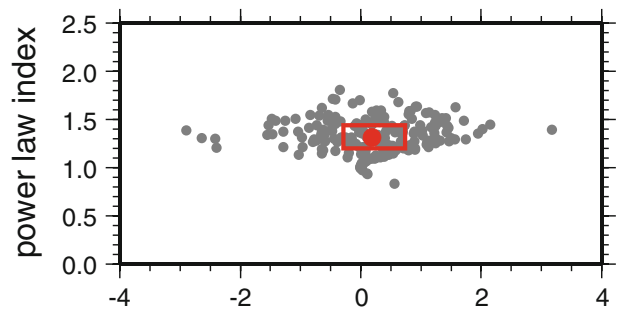

10 years RW

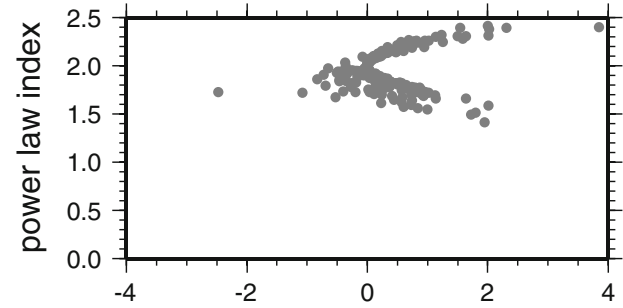

10 years FLRW

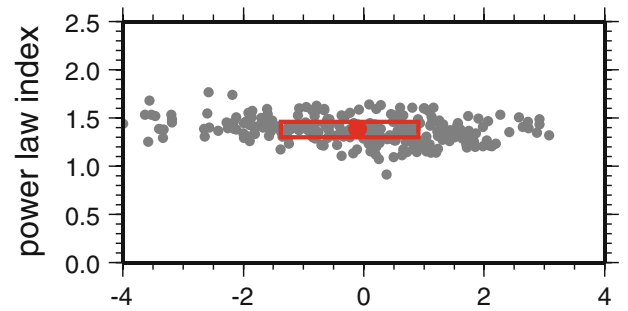

20 years $R W$

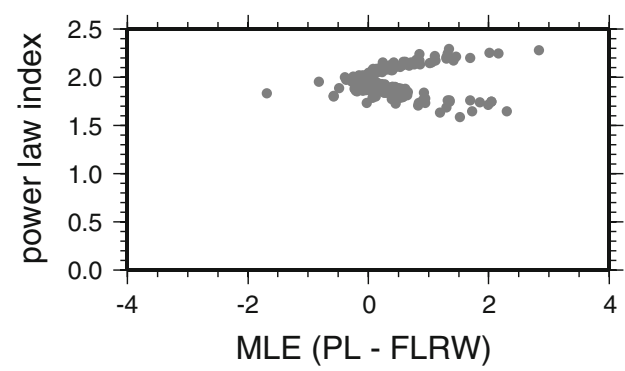

Fig. 3 Distribution of differences between $\log \left(\mathrm{MLE}_{\mathrm{PL}}\right)-\log$ (MLEFLRW) and the power-law index for the case where the underlying time series is RW plus WN (a) and FLRW plus WN (b). Each dot represents the results from a single simulation of data that was used

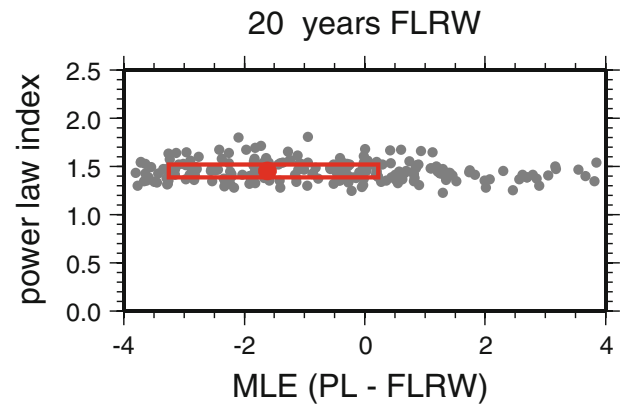

in Fig. 2. From each simulation, two different noise models were constructed: one being PL and the other being FLRW. The red dot and red boxes in (b) indicate the median and interquartile range for differences in likelihood and power-law index 
On the other hand, if the underlying data include a significant amount of FL noise, estimating the rate uncertainty reveals substantial bias (Fig. 2b). Discounting the shorter intervals, in particular the 1- and 2-year intervals, the rate uncertainty estimated using PL noise is about one half of the rate uncertainty estimated using the FLRW assumption for noise.

Unfortunately, for the intervals $\geq 5$ years, using the difference in the logarithm of the likelihood function does not provide guidance for which of the two models is better. Both models have three parameters, including the WN amplitude. Langbein (2004) introduced a method to test the difference in $\log$ (MLE) between two noise models, where the null hypothesis noise model has $N$ unknowns and the alternative has $N+1$ unknowns. To reject the null hypothesis in favor of the alternate model at the $95 \%$ level, $\delta \log$ (MLE) needs to exceed 2.6. In our case here, since both noise models have the same number of parameters, this statistical test is not valid. Nonetheless, it can provide a rough guide to distinguish between two competing models, for which these examples show that neither model is necessarily better since the $\delta \log (\mathrm{MLE})$ cluster around zero.

However, as the time series becomes longer, in excess of 20 years, there is a tendency for the differences to suggest that the FLRW model is a better description of the underlying distribution (Fig. 3b, bottom panel): that is, $\left|\log \left(\mathrm{MLE}_{\mathrm{PL}}\right)-\log \left(\mathrm{MLE}_{\mathrm{FLRW}}\right)\right|>2$.

The other subtle change as the length of the data set becomes longer is that the PL index becomes larger (Fig. 3b). For the shortest lengths, the PL index has a bimodal distribution with peaks at 1.5 and 0.5 . However, when the data length is 5 years in this set of simulations, the median index is 1.31 , and with 20 years, the median index increases to 1.45 .

\section{Estimating the random-walk component}

Assuming that FLRW noise is the underlying model of GPS time series, it can be difficult to reliably estimate long-period RW amplitude. This problem is frequently found with GPS time series of vertical position changes. In these data, both the WN and the FL amplitudes are typically a factor of two to three times larger than those found in the horizontal. Consequently, if the RW amplitude for the vertical is approximately the size found for the horizontal, the FL noise will dominate the spectrum over the limited observation period. Pictorially, this is the situation in Fig. 1 with only a year of data available. However, as Fig. 1 demonstrates, while detecting RW noise is difficult, its presence will impact the estimate of rate uncertainty.

In the absence of resolvable RW noise, there are three alternatives to pursue: (1) for vertical time series, assume that the RW amplitude estimated for the horizontal data cor- rectly describes the vertical, too; (2) assume that the RW noise is a consequence of monument wobble and use the RW amplitude determined from other studies; or (3) estimate the upper bound on the RW amplitude. Although none of these alternatives is a satisfactory solution, I will provide some guidance to estimate the upper bound for the RW noise.

The approach to obtain an upper bound on the RW amplitude is illustrated in Fig. 4, where the amplitude of RW noise, as an input to the MLE algorithm, is successively incremented and constrained to be above its optimal value. RW noise is incremented until the value of the logarithm of the likelihood function decreases to a value at which the noise model can be rejected at a pre-set confidence level, for example $95 \%$. In the example of using 1 year of data having the same FLRW model discussed previously, most of the estimates of optimal RW amplitude are $0 \mathrm{~mm} /$ year $^{0.5}$ (Fig. 4a), with $\delta \log (\mathrm{MLE})=0$. Increasing the value of RW noise to $0.2 \mathrm{~mm} /$ year $^{0.5}$ and fixing it to that value, then estimating the FL and WN amplitudes will yield a $\log$ (MLE) that is slightly less than that obtained for the optimal noise model. Successively increasing the RW noise yields individual curves with the $\log (\mathrm{MLE})$ decreasing from the optimal model. When the difference between $\log (\mathrm{MLE})$ and $\log \left(\mathrm{MLE}_{\text {optimal }}\right)$ is less than -2.6 , then the value of RW noise can be rejected at the $95 \%$ confidence level relative to the optimal model.

For the 20 simulations shown, 19 of the simulations have estimated RW amplitudes between 1.7 and $5 \mathrm{~mm} /$ year $^{0.5}$ when $\delta \log (\mathrm{MLE})=-2.6$; these values exceed the underlying RW amplitude of $1.3 \mathrm{~mm} /$ year $^{0.5}$. With each trial of RW noise, the rate uncertainty is also calculated (Fig. 4b). Correspondingly, with a rejection threshold of $\delta \log (\mathrm{MLE})=$ -2.6 , the upper bound of rate uncertainty ranges up to $5 \mathrm{~mm} /$ year, well in excess of the underlying value of $1.6 \mathrm{~mm} /$ year.

The same set of simulations is repeated for a 10-year long time series (Fig. 4c, d). With the longer time series, the upper bound on RW amplitude ranges between 1.5 and $2.3 \mathrm{~mm} /$ year $^{0.5}$, much closer to the underlying value of RW noise. In addition, the upper bound on the rate uncertainty is within a factor of two of the underlying uncertainty. The tighter bound with the longer data is not surprising, as shown in the power spectrum (Fig. 1) as RW noise becomes resolvable.

\section{Discussion and conclusions}

Motivation for this study was provided by the observation of Langbein (2008) that the background noise model for many GPS time series can be either FLRW or PL with the powerlaw index ranging between 1 and 2 . However, the choice of noise model will impact the estimate of the rate uncertainty or any other parameter derived from the data that relies upon a long period of observation. The dependence of rate 

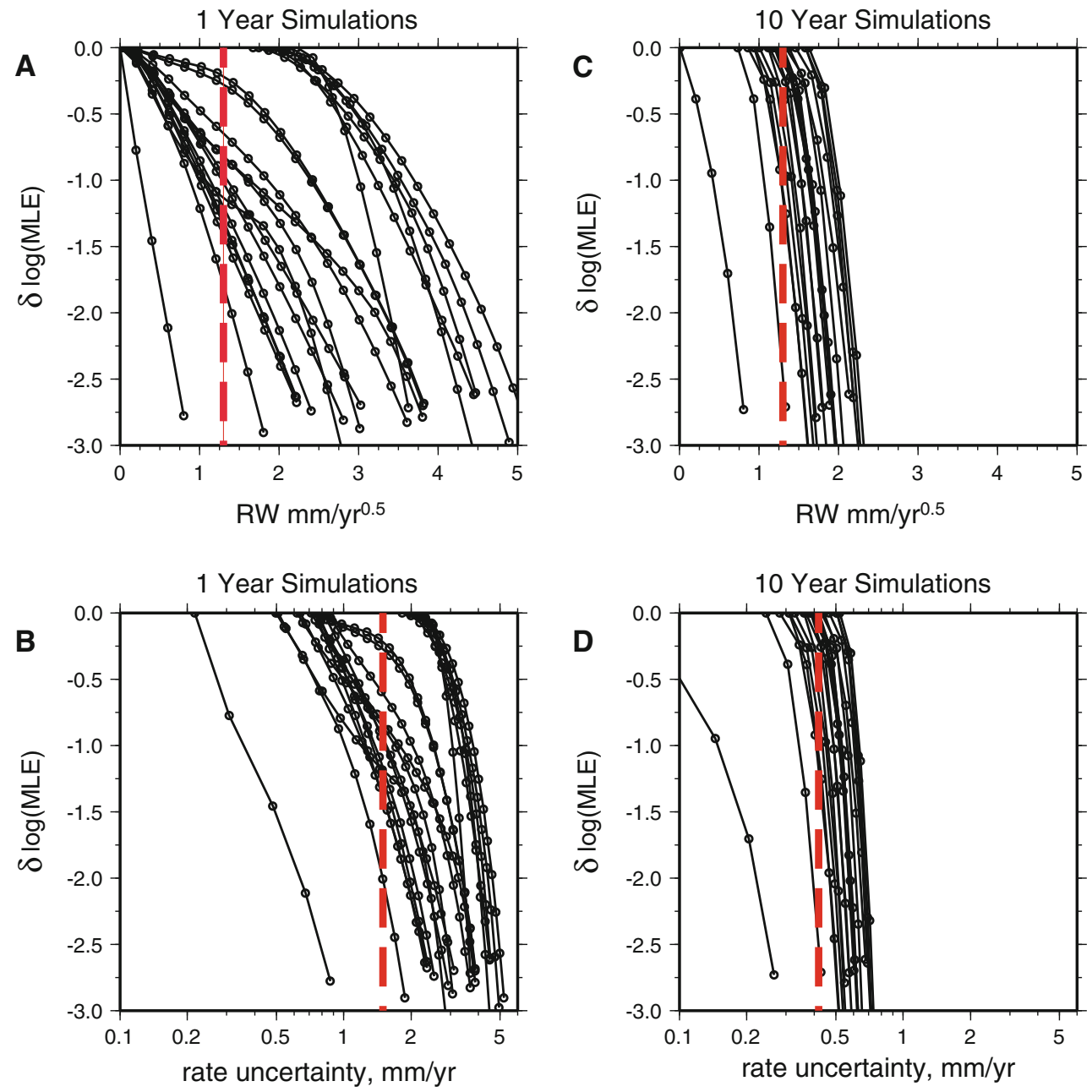

Fig. 4 Determination of the upper bound for RW noise and the corresponding uncertainty in rate for 20 simulations for two different data lengths, 1 and 10 years. The underlying noise model for all simulations is the same as Fig. 1. a Each curve represents a single, simulated data set of 1 year in length showing the change in $\log$ (MLE) from the optimal value as the amount of RW noise is increased in increments of $0.2 \mathrm{~mm} /$ year $^{0.5}$. The vertical dashed line is the underlying RW noise

$\left(1.3 \mathrm{~mm} /\right.$ year $\left.^{0.5}\right)$. b The uncertainty in rate for each simulation as the RW noise is increased. The vertical dashed line is the rate uncertainty associated with the underlying noise model. c, $\mathbf{d}$ This is the same computation as $\mathbf{a}$ and $\mathbf{b}$, but with the interval increased to 10 years. Note that the rate uncertainty is smaller than the 1-year case since the interval is longer

uncertainty with the choice of noise model is illustrated in Fig. 2, with the uncertainty estimated with a PL model being about one half of the uncertainty estimated with a FLRW model.

The choice between these two models can be decided by two ways. The more conservative approach is to accept that monument wobble is present in the data and the noise for that process is a random walk similar to the noise characteristics found in other crustal deformation measuring instruments, primarily strainmeters and creepmeters, which use transducers that are more sensitive to displacements than GPS.

However, after evaluating a GPS network and modeling the rates for a physical mechanism of deformation of the network, one obtains a measure of fit of the physical model to the data. That fit is characterized as the difference between

the predicted rates and the observed rates normalized by the rate uncertainty, and is termed the misfit ratio. If one had used the FLRW noise model and obtained a misfit ratio much less than unity, it is reasonable to conclude that the FLRW model of noise is much too conservative or that the model of deformation is too complex for the quantity and quality of the observations. Assuming the data were not over fit with a complex model, one could reasonably conclude that the PL noise model is the more appropriate choice. However, more often than not, the misfit ratio is greater than unity suggesting that the more conservative noise model might be more appropriate or that the assumptions for the physical model are incorrect.

The other problem due to a combination of high noise and short time series is that the long-period component of noise is 
not well resolved. The result is that the long-period component is underestimated and the corresponding rate uncertainty is too small. An extreme example is data from campaign-style GPS, where the observations might be made at yearly intervals. Here, the sampling rate is just too low and precludes any quantitative estimate of the noise spectra. Consequently, one often makes an "educated guess" at the noise components.

The ability to resolve the longer-period parts of the noise spectrum depends greatly on the level of noise over the shorter periods. At its most basic level, this was discussed by Langbein and Johnson (1997) and they characterized the problem in terms of the crossover frequency, where power from RW noise equals that of WN. In addition, to resolve the RW component, they stated that the period representing the crossover frequency should be less than one tenth of the total time span of the data. With many GPS time series with time spans in excess of 10 years, the crossover between WN to the longer-period noise is well resolved (Fig. 1). What is less clear though, is the character of the longer-period noise. Is the slope of the noise spectrum in log-log space constant, with either the slope having an integer value representing FL or RW noise, or a constant, non-integer value? Or, does the slope change with a secondary crossover period that is longer than the crossover to WN? Is that secondary crossover at a period longer than the time span of the data?

After examining the potential biases potentially built into the fact that there will never be enough data to accurately assess the error spectrum, I recommend that error assessment be carried out as follows:

Remove outliers and bad data. By removing outliers, this decreases the amount of measured $\mathrm{WN}$ and provides better resolution of the crossover between the white noise and the power-law part of the noise spectrum. Outliers can be either identified visually, or after using a robust data-smoother such as a running median to remove the long-period trends, then deleting those observations that exceed a specified amount. Alternatively, Khodabandeh et al. (2011) suggest another means to mitigate for the presence of the occasional outlier. At the extreme, there is a possibility that excessive white noise could mask completely the underlying, longer-period noise, and because the computed rate uncertainty decreases with the number of observations under the assumption of uncorrelated data, the estimate of uncertainty will be less than the true uncertainty.

Identify offsets, rate changes, periodicities, etc. The presence of any of these features in the data has the potential to contribute to the noise spectrum at the longer periods. Construction of periodograms can help in identifying periodic components in the data. Sometimes, it is required that one alternately identify and remove the various functional depen- dencies and go back and remove outliers and bad sections of data.

Estimate the noise spectrum. Either a maximum-likelihood technique of fitting trial data covariances to the data or spectral techniques working in the frequency domain can be employed to quantify the background noise. With MLE techniques, both the parameters of the time dependence identified in the second step and the parameters of the modeled data covariance are estimated simultaneously. It should be emphasized, especially based upon the work presented above, that several different models should be tried and tested (Langbein 2004). With spectral techniques and the caveat that the results can depend upon the window length and the tapers employed, the results should be plotted and analyzed to extract simple functions that represent the noise power as a function of frequency. By extracting the parameters representing a noise spectrum, the data covariance can be constructed using Eq. 10 of Williams (2003). Spectral techniques have the advantage of being computationally faster than MLE techniques, but they are restricted to data with no gaps in observations, although adequate spectra can be estimated for data with a few, short gaps in observations.

This paper discusses only PL and FLRW noise, but as Davis et al. (2012) demonstrate, seasonal noise having variable amplitude and phase can be a broad-band signal that, if not recognized, can corrupt any inference obtained if the PL or FLRW noise models are the only models evaluated. Davis et al. (2012) recommend filtering the data, but Langbein (2004) provides methods to accommodate the variable nature of seasonal noise into the data covariance. Thus, not only should one evaluate the periodicities that have non-time varying amplitudes and phase along with offsets and rate change, other noise models using bandpass filtered noise (Langbein 2004) should be evaluated along with the basic PL or FLRW models. Choosing the most appropriate model is done by comparing the $\log (\mathrm{MLE})$ of the competing noise models.

Assessment of noise model. If the MLE method is employed to estimate the noise model, then the equivalent power density spectrum should be plotted using Eq. 10 of Williams (2003). If the results suggest that the long-period noise is best characterized by FL noise or PL with an index that is significantly less than 2 , then the absence of RW noise needs to be evaluated in terms of whether the amplitude of FL precludes the detection of the RW component, or the time span of the data is just too short to extract a RW component. Comparison with other data, such a multiple, long time-series of GPS data, could suggest that the RW component is not present. In addition to plotting a power density spectrum, variograms (Agnew 1992) should be constructed for the data along 
with simulations of the data using the noise model of the data. A variogram is a statistical measure of the mean-square difference of the current observation from previous observations as a function of the time interval between the observations. With white noise, the variogram has a constant value, but with power-law noise, the level of the variogram increases with longer intervals.

Examination of the noise characteristics of short-baseline GPS time series does suggest that RW noise is present in these data (Hill et al. 2009; King and Williams 2009). Analysis of a 33-m long baseline using braced monuments, which are considered best practice, by King and Williams (2009) indicates that the RW noise could be at the $0.5 \mathrm{~mm} /$ year $^{0.5}$ level, or a factor of three less than the example used in this paper. This conclusion is supported by Hill et al. (2009) in their examination of another set of short baselines ranging between $10 \mathrm{~m}$ and $1 \mathrm{~km}$ using braced monuments. Analyzing short baselines has the advantage of removing a number of common-mode sources when estimating position of one site relative to another. Consequently, the position estimates from short baselines tend to have small $\mathrm{WN}(<0.2 \mathrm{~mm})$ in their observations relative to the position estimates obtained from regional networks (e.g. King and Williams 2009).

Further assessment of the reliability of the noise model can be accomplished by obtaining the maximum value of RW noise allowed by the data using the method outlined in Fig. 4. Whether that constraint is useful or not is another question. More likely, the upper bound will grossly exceed the underlying value of RW noise. Consequently, one should compare the upper bound on the RW noise with that from the analysis of King and Williams (2009) for short baselines.

The basic message is contained in Fig. 2. The choice of noise model can bias the estimate of uncertainty. It is best to use long periods of data to extract the estimates of noise at the longest periods. The most conservative approach in terms of estimating GPS velocities would be to use the FLRW model to represent noise in GPS time series of positions. With the current techniques available, it is usually not possible to distinguish between the two most likely models of GPS noise, PL and FLRW. Instead, one should examine other sources of information, including whether other GPS data have similar site characteristics, and other environmental factors that might affect the type of noise.

Open Access This article is distributed under the terms of the Creative Commons Attribution License which permits any use, distribution, and reproduction in any medium, provided the original author(s) and the source are credited.

\section{References}

Agnew D (1992) The time domain behavior of power law noises. Geophys Res Lett 19(4): 333-336. doi:10.1029/91GL02832

Amiri-Simkooei AR, Tiberius CCJM, Teunissen PJG (2007) Assessment of noise in GPS coordinate time series: methodology and results. J Geophys Res 112. doi:10.1029/2006JB004913

Beavan J (2005) Noise properties of continuous GPS data from concrete pillar geodetic monuments in New Zealand and comparison with data from U.S. deep drilled braced monuments. J Geophys Res 110:B08410. doi:10.1029/2005JB003642

Calais E, Stein S (2009) Time-variable deformation in the New Madrid Seismic zone. Science 323:1442

Davis JL, Wernicke BP, Tamisiea ME (2012) On seasonal signals in geodetic time series. J Geophys Res 117:B01403. doi:10.1029/ 2011JB008690

Frankel A, Smalley R (2011) Significant motion between GPS sites in the New Madrid region; implications for seismic hazard. Bull Seismol Soc Am (submitted)

Hill EM, Davis JL, Elósegui P, Wernicke BP, Malikowski E, Niemi NA (2009) Characterization of site-specific GPS errors using short-baseline of braced monuments at Yucca Mountain, southern Nevada. J Geophys Res 114:B11402. doi:10.1029/2008JB006027

Hough SE, Page M (2011) Toward a consistent model for strain accrual and release for the New Madrid Seismic Zone, Central United States. J Geophys Res 116. doi:10.1029/2010JB007783

Johnston MJS, Linde AT (2002) Implications of crustal strain during conventional, slow, and silent earthquakes. In: Lee WHK (ed) et al International Handbook of Earthquake Engineering and Seismology, vol 81A, chap 36. Int Assoc Seismol Phys Earths Interior, pp 589-605

King MA, Williams SDP (2009) Apparent stability of GPS monumentation from short-baseline time series. J Geophys Res 114:B01403

Khodabandeh A, Amiri-Simkooei AR, Sharifi MA (2011) GPS position time-series analysis based on asymptotic normality of M-estimation. J Geod 85 doi:10.1007/s00190-011-0489-4

Langbein J, Breckenridge K, Quilty E (1993) Sensitivity of crustal deformation instruments to changes in secular rate. Geophys Res Lett 20(2):85-88

Langbein J, Johnson H (1997) Correlated error in geodetic time series: implications for time-dependent deformation. J Geophys Res 102:591-604

Langbein J (2004) Noise in two-color electronic distance meter measurements revisited. J Geophys Res 109:B04406. doi:10.1029/ 2003JB002819

Langbein J (2008) Noise in GPS displacement measurements from Southern California and Southern Nevada. J Geophys Res 113:B05405. doi:10.1029/2007JB005247

Mao A, Harrison CGA, Dixon TH (1999) Noise in GPS coordinate time series. J Geophys Res 104:2797-2816

Santamaría-Gómez A, Bouin MN, Collilieux X, Wöppelmann G (2011) Correlated errors in GPS position time series: implications for velocity estimates. J Geophys Res 116. doi:10.1029/ 2010JB007701

Wdowinski S, Bock Y, Zhang J, Fang P, Genrich J (1997) Southern California permanent GPS geodetic array: spatial filtering of daily positions for estimating coseismic and postseismic displacements induced by the 1992 Landers earthquake. J Geophys Res 102(B8): 18057-18070

Williams SDP (2003) The effect of coloured noise on the uncertainties of rates estimated from geodetic time series. J Geod 76: 483-494. doi:10.1007/s00190-002-0283-4 
Williams SDP, Bock Y, Fang P, Jamason P, Nikolaidis RM, Prawirodirdjo L, Miller M, Johnson DJ (2004) Error analysis of continuous GPS position time series. J Geophys Res 109. doi:10.1029/ 2003JB002741

Wyatt F (1982) Displacement of surface monuments: horizontal motion. J Geophys Res 87:979-989. doi:10.1029/ JB087iB02p00979
Wyatt FK (1989) Displacement of surface monuments: vertical motion. J Geophys Res 94:1655-1664. doi:10.1029/JB094iB02p01655

Zhang J, Bock Y, Johnson H, Fang P, Williams S, Genrich J, Wdowinski S, Behr J (1997) Southern California permanent GPS geodetic array: error analysis of daily position estimates and site velocities. J Geophys Res 102(B8):18035-18055 doi:10.1029/97JB01380 